\title{
Vehicle Cartoon Voice Intelligent Driving Control System
}

\author{
Jianchun Gong*, Yuhai Jiang and Yuanxue Tao \\ Panzhihua University, Sichuan, 617000, P.R. China \\ ${ }^{*}$ Corresponding author
}

\begin{abstract}
This paper mainly introduces the intelligent control system of vehicle's voice driving, and designs a complete set of automotive language - animation language. With this language as the medium, the communication between the driver and the intelligent vehicle is realized so as to achieve the intelligent driving of the car. Thereby achieving the aim of reducing or even eliminating various conventional control operations.
\end{abstract}

Keywords-voice; anime language; communication for information; medium; for intelligent driving; Medium

\section{INTRODUCTION}

In recent years, with the rapid development of automobile industry and the continuous improvement of human quality of life, automobile has gradually become a necessity of human beings. At the same time, a variety of functions can meet the needs of people's lives are also increasing. This poses a very high requirement for the driver's driving technology. If you have a common voice, you can manipulate the communication between the driver and the car that guides the car. As if the Chinese communicate with Chinese people through Chinese, foreigners can also communicate with Chinese by learning Chinese. When this equivalent information exchange between the car and the driver can be carried out, the driver can drive in a more comfortable and harmonious environment. Therefore, the design of such a specific animation language between the driver and the car, as a medium, the intelligent control system between the driver and the vehicle will be connected together, will greatly improve the driver driving control environment. Improve operation efficiency.

\section{THEORETICAL BASIS}

Sound control is a simple explanation of the system, and speech recognition is one of its core technologies. At present, the latest speech recognition technology can recognize more than $90 \%$ words spoken by humans. The intelligent control system of the automobile can receive the human communication control information in time and accurately, and make the corresponding judgment, so that the control task can be completed independently. Is the biggest challenge it faces.

Because there are too many influencing factors in the actual operation environment for voice transmission, the quality of voice control technology in wireless transmission is not very good, which will directly affect the accuracy of the information reception of the controller. Animation speech intelligent system to deal with this way: $\mathrm{C}$ language programming preprocessing program. For each control instruction ' s voice input, accept in the form of keyword, through spce061a single chip ( Lingyang technology ) a 16 - bit structure microcontroller, has higher processing speed, can process complex digital signal quickly ; Has programmable audio processing; built-in 2kword sram and

$32 \mathrm{kword}$ flash; Speech processing for two 16-bit programmable timers / counters (automatic preset initial count values, 2 10-bit dac output channels, 32-bit general-purpose programmable input / output ports). The characteristics of different instructions, corresponding to the preparation of a single, no repetition of the preprocessing program. Of course, it is necessary to write a huge preprocessor Library in advance to facilitate the call of the main function when the characteristics of different instructions appear. As shown in figure 1.

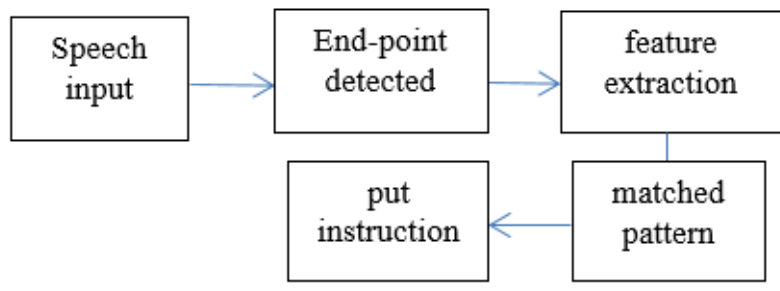

Voice recognition, control system principle flow diagram

FIGURE I. SPEECH RECOGNITION FLOW CHART

Since speech recognition can be divided into single words input when the driver is operating according to the actual need. For example: drive, turn left, slow down, etc. And there's a continuous voice input. For example, please drive, please open the air conditioner, please open the window, etc.. Like the human language world, different words, different numbers of words, together in the meaning of expression will be different. In the currently applied speech recognition system, it is more important to use the matching of the characteristic instruction to call the characteristic function instruction to control, so as to realize the specific function. While the voice control system according to the Chinese characters dictionary pronunciation animation, different Chinese characters to audio digital signal of different pre match options included, as alternative pretreatment instructions, using 61 single-chip voice information docking high speed fast by the recognition process.

For example, simple forward subroutine, reverse subroutine, left and right turn subroutine and so on. After compiling the program language, it is the preparation of the hardware. The 
vehicle body model of the intelligent vehicle is primarily used, including power supply module, motor drive module, circuit board, etc. When the circuit is connected, the 61 single chip computer is loaded and the initial voice test is carried out.

\section{EXPERIMENTAL SCHEME}

With Lingyang spce061a 16 - bit single - chip microcomputer, the whole driving process of the trolley is monitored in real time. Since the single chip of Lingyang spce061a provides $32 \mathrm{i} /$ o ports, all functions can be realized, which provides great convenience for the design process. The main design idea is: install a Holzer element on the car, use the external interrupt of iob3 to identify the result of tire rotation, and use it to calculate the distance and use led to display it. Three photodetectors for detecting obstacles and a collision switch are installed, and the turning is controlled by means of scanning iob4, iob5, iob6; using the iob8-iob14 control relay of single chip computer to select the positive, reverse and add, slow down. The voice command adopts wireless receiving module to carry on the speech remote control. The response speed and accuracy of the control system were recorded by the experiment of different populations of speech to the same command. Also can use different motor, gear transmission and other hardware structure. According to the results, the effects of external factors such as hardware on the experimental data are eliminated.

Of course, at present, in the field of voice control, the main principles of speech recognition are divided into two stages: "training" and "recognition". In the training stage, the singlechip carries out the analysis and processing of the collected voice samples, extracts the speech feature information and establishes a feature model. In the recognition stage, the singlechip performs similar analysis processing on the collected voice samples, extracts the characteristic information of the speech, and then compares the characteristic information model with the existing feature model, and if the matching degree is reached, the input speech is recognized. The difference between this control principle is that the anime voice intelligent system has changed its training stage, which has become previously included or called early preparation. The difference between this control principle is that the anime voice intelligent system has changed its training stage, which has become previously included or called early preparation. The invention has the advantages that the characteristic model can be avoided during the training phase. This results in the delay of receiving information and even the decrease of accuracy in the recognition stage. This reduces the responsiveness of the control system. It's like a well-prepared interviewee who can handle the interviewer's questions at a leisurely pace.

\section{COMPARISON}

Voice intelligent control system compared with the previous intelligent control system. First of all, human language vocabulary must be recorded as an audio digital signal corresponding to a single word. We can follow the modern edition of the Chinese Dictionary or the English Dictionary, of course, this is a very large preparatory work. But once it's done, it's like the chef has prepared the main ingredients. In the cooking operation, you can according to the order of the operation, come at once. Secondly, every operating command of the car has a fixed characteristic command. For example, "start" is an operation command, or a characteristic signal, which is composed of two audio digital signals, starting and moving, in order of sequence. When our single-chip computer receives this signal, it will send out the control command according to the set-up program, so as to control the car to make the correct action. Therefore, writing a preprocessing program composed of feature signals is also a high-intensity preparatory work. To complete the operation of the car can be completed, prepared in advance.

Compared with other intelligent control systems, although the preparatory work in the early stage of this project is very large, in the later testing process, the sensor receives the voice signal and the singlechip to extract the characteristic signal and the processing speed is more convenient. And the environmental interference degree of the characteristic signal is greatly reduced in the process of transmission. The whole process is like reading a dictionary. And each execution command is a statement that consists of a single 'word' or a single 'sound'. The command issued by the driver, through this phonetic system, can quickly match the execution of the command. Thus, voice driving is realized. Of course, the speed of speech matching should be determined by hardware configuration, Lingyang spce061a 16 bit MCU is not necessarily the most appropriate. The most appropriate hardware configuration is determined by a considerable amount of experimental testing.

If intelligent driving is regarded as an excellent dish, audio recording and preprocessing are the most important ingredients preparation, while other hardware devices are like seasoning. As we all know, seasoning determines the taste of the food and changes it according to personal taste. In other words, the intelligent voice driving system has entered the final debugging stage. Through a lot of tests, you can perfect it to a satisfactory and even unexpected height.

\section{CONCLUSIONS}

The present age, is precisely the automobile industry highspeed development time. The intelligent automobile is a new field that the automobile is about to enter, and it is a significant reform of the automobile industry. It is believed that the "animation and voice intelligent driving control system" will become a new star in the age of smart cars.

\section{REFERENCES}

[1] Jie Li and Li Huaiyu, Discussion on the value of used car in accident, Academic research of Shijiazhuang Polytechnic University, Career Academy,2016.

[2] Zhao Mengying, Research on the development of second-hand car market in China. Master's degree thesis of Huazhong Normal University, 2012.

[3] Xiao Huan. Study on depreciation regulation of used cars. Journal of Chifeng University (NATURAL SCIENCE EDITION) 2015.06.027

[4] Zhang Lijun. Research on American used car market model. Shanghai automobile, 2010.

[5] Into English, Liu Xiaofeng, Wang Lei, Wang Nongzhi. Automobile evaluation. Tsinghua University press, 2016.

[6] Jian-Da, Wu, Chuang-Chin, Hsu, Hui-Chu, Chen., An, expert, system, of, price, forcasting, for, used, cars, using,, adaptive, neuro-fuzzy, inference., Expert, application, systems, with,... 
[7] Peng Jianglei. Study on current market price method for second-hand car evaluation. Master's degree thesis of Nanjing University of Science and Technology, 2009.

[8] Huang Qiuping. Selection and comparison of appraisal methods for used cars. Traffic world, 2012.

[9] Wang Qin, Wu Kelin, Du Shidong, Liang Hongming. Replacement cost method for value assessment of used cars. Automotive and accessories, 2010.

[10] Pang, Changle. Practice and evaluation of used car. First edition. Beijing Institute of Technology press, 2007.

[11] Yu Yu. Automotive theory. Fifth edition. China Machine Press, 2015.

[12] Wang Zhihong, Liu Chengwu. Automotive inspection and maintenance. First edition. People Communications Press, 2013.

[13] Yang Yuanlei. Research on the evaluation method of old motor vehicle based on the replacement cost method [D]. Chang'an University, 2012. 